\title{
Differential equation for the Dirac single-particle first-order density matrix in terms of the ground-state electron density
}

\author{
N. H. March, ${ }^{1}$ T. Gál, ${ }^{2}$ and I. A. Howard ${ }^{3}$ \\ ${ }^{1}$ Department of Physics, University of Antwerp, B-2020 Antwerp, Belgium, and Oxford University, Oxford, UK \\ ${ }^{2}$ Section of Theoretical Physics, Institute of Nuclear Research of the Hungarian Academy of Sciences, H-4001 Debrecen, Hungary \\ ${ }^{3}$ Department of Chemistry, Free University of Brussels (VUB), B-1050 Brussel, Belgium
}

(Received 9 October 2009; published 22 June 2010)

\begin{abstract}
The March-Suhai (MS) partial differential equation for the Dirac density matrix $\gamma_{s}\left(\vec{r}, \vec{r}^{\prime}\right)$, proved for oneand two-level occupancies, involves both the ground-state density $n(\vec{r})$, with its low-order derivatives, and the positive definite kinetic energy density $t_{s}(\vec{r})$. Here, we examine the relation between the equation of motion for $\gamma_{s}\left(\vec{r}, \vec{r}^{\prime}\right)$, with input now being the one-body potential of density-functional theory, and the MS equation. The important link is the differential virial theorem, which can be used to remove $t_{s}(\vec{r})$ from the MS differential equation. For multiple occupancy, the Pauli potential enters in an important manner. In one dimension, however, the appearance of the Pauli potential can be avoided, obtaining a necessary condition for $\gamma_{s}\left(x, x^{\prime}\right)$ to satisfy for arbitrary level occupancy, in the form of a MS-type differential equation.
\end{abstract}

DOI: 10.1103/PhysRevA.81.064503

PACS number(s): 31.15.E-, 71.15.Mb

\section{BACKGROUND}

March and Suhai (MS) [1] recently raised the question as to whether the Dirac single-particle first-order density matrix $\gamma_{s}\left(\vec{r}, \vec{r}^{\prime}\right)$ [2], within the framework of current formulations of density-functional theory (DFT) [3], could be determined with input of the ground-state density $n(\vec{r})$ determined experimentally from x-ray diffraction studies. They gave a partially affirmative answer, by setting up a differential equation for $\gamma_{s}\left(\vec{r}, \vec{r}^{\prime}\right)$ for one- and two-level occupancy (e.g., He and $\mathrm{H}_{2}$ for one level and Be and $\mathrm{LiH}$ for two levels), with $n(\vec{r})$ and the single-particle kinetic energy density $t_{s}(\vec{r})$ as input, where the latter is an (unknown) functional of $n(\vec{r})$. Here, we show that a differential equation paralleling the MS equation can be derived for $\mathrm{He}$ and $\mathrm{Be}$. With the use of this equation, the kinetic energy term can be completely eliminated from the MS equation, obtaining an explicit equation for the Dirac density matrix as a functional of the density. In one dimension, it will further be shown that a MS-type equation for $\gamma_{s}\left(x, x^{\prime}\right)$ can be set up for arbitrary level occupancy. With insertion of the known expression for $t_{s}[n](x)$ for noninteracting electrons in harmonic confinement into this equation, an explicit differential equation for $\gamma_{s}[n]\left(x, x^{\prime}\right)$ is obtained for this case.

From $\gamma_{s}\left(\vec{r}, \vec{r}^{\prime}\right)$, the single-particle kinetic energy density,

$$
t_{s}(\vec{r})=\left.\frac{\hbar^{2}}{2 m} \nabla_{\vec{r}^{\prime}} \nabla_{\vec{r}} \gamma_{s}\left(\vec{r}^{\prime}, \vec{r}\right)\right|_{\vec{r}^{\prime}=\vec{r}},
$$

and the exchange energy density,

$$
\epsilon_{x}(\vec{r})=-\frac{e^{2}}{4} \int \frac{\gamma_{s}\left(\vec{r}, \vec{r}^{\prime}\right) \gamma_{s}\left(\vec{r}^{\prime}, \vec{r}\right)}{\left|\vec{r}-\vec{r}^{\prime}\right|} d \vec{r}^{\prime},
$$

can be calculated. The latter of course determines the corresponding Slater exchange potential [4], $V_{x}^{\text {Slater }}(\vec{r})=\frac{2 \epsilon_{x}(\vec{r})}{n(\vec{r})}$, too, which importantly contains correctly the self-interaction correction. This requires that far from all nuclei, the correct exchange potential $V_{x}(\vec{r}) \rightarrow-\frac{e^{2}}{r}$, and this condition is readily shown to follow from the asymptotic form of $\epsilon_{x}(\vec{r})$ [5], namely,

$$
\epsilon_{x}(\vec{r}) \underset{|\vec{r}| \rightarrow \infty}{\rightarrow}-\frac{e^{2}}{2 r} n(\vec{r}),
$$

obtained using the idempotency of the Dirac density matrix.

\section{DIFFERENTIAL EQUATION FOR THE DIRAC DENSITY MATRIX, GIVEN THE GROUND-STATE ELECTRON DENSITY AS INPUT}

For one- and two-level occupancy, March and Suhai [1] showed that the Dirac single-particle first-order density matrix $\gamma_{s}\left(\vec{r}^{\prime}, \vec{r}\right)$ satisfies the differential equation

$$
\begin{aligned}
& {\left[\nabla_{\vec{r}} \gamma_{s}\left(\vec{r}, \vec{r}^{\prime}\right)\right]^{2}-\gamma_{s}\left(\vec{r}, \vec{r}^{\prime}\right) \nabla_{\vec{r}} \gamma_{s}\left(\vec{r}, \vec{r}^{\prime}\right) \frac{\nabla_{\vec{r}} n(\vec{r})}{n(\vec{r})}+2 \frac{t_{s}(\vec{r})}{n(\vec{r})}} \\
& \quad \times\left[\gamma_{s}\left(\vec{r}, \vec{r}^{\prime}\right)\right]^{2}-2 n\left(\vec{r}^{\prime}\right)\left\{t_{s}(\vec{r})-\frac{1}{8} \frac{\left[\nabla_{\vec{r}} n(\vec{r})\right]^{2}}{n(\vec{r})}\right\}=0,
\end{aligned}
$$

in atomic units. From the fundamentals of DFT, it follows that $t_{s}(\vec{r})$ is a functional of $n(\vec{r})$; hence, Eq. (4) relates $\gamma_{s}\left(\vec{r}^{\prime}, \vec{r}\right)$ solely to $n(\vec{r})$, with no one-body potential $V(\vec{r})$ involved. It is worth noting that the expression in the curly braces in Eq. (4) is just the Pauli kinetic energy density (i.e., the correction to the Weizsäcker single-particle kinetic energy density due to the exclusion principle for fermions).

Alternatively, with $V(\vec{r})$ as the input, we have the equation of motion [6],

$\nabla_{\vec{r}}^{2} \gamma_{s}\left(\vec{r}, \vec{r}^{\prime}\right)-\nabla_{\vec{r}^{\prime}}^{2} \gamma_{s}\left(\vec{r}, \vec{r}^{\prime}\right)=\frac{2 m}{\hbar^{2}}\left[V(\vec{r})-V\left(\vec{r}^{\prime}\right)\right] \gamma_{s}\left(\vec{r}, \vec{r}^{\prime}\right)$,

for $\gamma_{s}\left(\vec{r}, \vec{r}^{\prime}\right)$, which is valid for arbitrary level occupancy. To connect Eqs. (4) and (5), we now appeal to the differential virial theorem of Holas and March [7]. This reads, in 
spherical symmetry (which we will focus on exclusively in the following), but for general level occupancy,

$$
-\frac{\partial V(r)}{\partial r}=\frac{z_{s}(r)}{n(r)}-\frac{\hbar^{2}}{4 m} \frac{1}{n(r)} \frac{\partial}{\partial r} \nabla^{2} n(r) .
$$

The quantity $z_{s}(r)$ is the radial component of the single-particle limit of the many-electron vector field $\vec{z}(\vec{r})$ defined in [7] from the kinetic energy density tensor $t_{\alpha \beta}(\vec{r})$,

$$
t_{\alpha \beta}(\vec{r})=\frac{\hbar^{2}}{4 m}\left[\frac{\partial^{2}}{\partial r_{\alpha} \partial r_{\beta}^{\prime}} \gamma\left(\vec{r}, \vec{r}^{\prime}\right)+\frac{\partial^{2}}{\partial r_{\beta} \partial r_{\alpha}^{\prime}} \gamma\left(\vec{r}, \vec{r}^{\prime}\right)\right]_{\vec{r}^{\prime}=\vec{r}},
$$

as

$$
z_{\alpha}(\vec{r})=2 \sum_{\beta=1}^{3} \frac{\partial t_{\alpha \beta}(\vec{r})}{\partial r_{\beta}}
$$

Dividing Eq. (5) by $\gamma_{s}\left(\vec{r}, \vec{r}^{\prime}\right)$, and then applying the gradient operator, with $\vec{r} \nabla=r \frac{\partial}{\partial r}$, we readily obtain

$$
\frac{\partial V(r)}{\partial r}=\frac{\hbar^{2}}{2 m} \frac{\vec{r}}{r} \nabla_{\vec{r}}\left[\frac{\nabla_{\vec{r}}^{2} \gamma_{s}\left(\vec{r}, \vec{r}^{\prime}\right)-\nabla_{\vec{r}^{\prime}}^{2} \gamma_{s}\left(\vec{r}, \vec{r}^{\prime}\right)}{\gamma_{s}\left(\vec{r}, \vec{r}^{\prime}\right)}\right],
$$

from which $\partial V / \partial r$ can be eliminated with the use of Eq. (6).

For He, it is known from the work of Akbari et al. [8] that the first term on the right of Eq. (6) can be written exactly in terms of the Weizsäcker kinetic energy density $t_{W}(\vec{r})$, defined by

$$
t_{W}(\vec{r})=\frac{\hbar^{2}}{8 m} \frac{[\nabla n(\vec{r})]^{2}}{n(\vec{r})} .
$$

Equation (6) becomes, for $\mathrm{He}$,

$-\frac{\partial V(r)}{\partial r}=\frac{4}{n(r)}\left[\frac{t_{W}(r)}{r}+\frac{1}{2} \frac{\partial t_{W}(r)}{\partial r}\right]-\frac{\hbar^{2}}{4 m} \frac{1}{n(r)} \frac{\partial}{\partial r} \nabla^{2} n(r)$,

which must be equivalent to the first derivative of the wellknown von Weizsäcker equation [9]. By eliminating $\partial V / \partial r$ by adding Eqs. (9) and (11), we get a differential equation for $\gamma_{s}\left(\vec{r}, \vec{r}^{\prime}\right)$, with the density $n(r)$ and the kinetic energy density $t_{W}(r)$ [with the known density dependence, Eq. (10)] as input, paralelling the March-Suhai equation, Eq. (4), in spherical symmetry, for one-level occupancy (such as in He-like atomic ions). Since for one-level occupancy, the Dirac density matrix is a known functional of the density,

$$
\gamma_{s}\left(\vec{r}, \vec{r}^{\prime}\right)=\sqrt{n(\vec{r})} \sqrt{n\left(\vec{r}^{\prime}\right)}
$$

the differential equation obtained can be explicitly tested, giving an identity when Eq. (12) is inserted.

In a recent study, March and Nagy [10] have generalized this result of Akbari et al. for arbitrary level filling in spherically symmetric atoms as

$$
z_{s}(r)=4\left[\frac{t_{W}(r)}{r}+\frac{1}{2} \frac{\partial t_{W}(r)}{\partial r}\right]+n(r) \frac{\partial V_{P}(r)}{\partial r},
$$

where $V_{P}(r)$ is the Pauli potential [11,12] (for the case of spherical symmetry), defined by

$$
V_{P}(\vec{r})=\frac{\delta T_{s}[n]}{\delta n(\vec{r})}-\frac{\delta T_{W}[n]}{\delta n(\vec{r})} .
$$

We note here that Eq. (13) is not an expression obtained from Eq. (8). Equation (6) with Eq. (13) inserted is simply the derivative, with respect to $r$, of the Euler equation of DFT, with Eqs. (10) and (14) utilized. That is, $V_{P}(r)$ enters Eq. (6) purely formally.

For the two-level case, one can use the Dawson-March form of the Dirac density matrix [13], namely,

$$
\gamma_{s}\left(\vec{r}, \vec{r}^{\prime}\right)=\sqrt{n(r)} \sqrt{n\left(r^{\prime}\right)} \cos \left[\theta(r)-\theta\left(r^{\prime}\right)\right]
$$

in terms of the density amplitude $n^{\frac{1}{2}}(r)$ and the phase factor $\theta(r)$. The phase factor $\theta(r)$ can be determined as a functional of the density from a nonlinear pendulum equation into which the density is the sole input; with $\theta[n]$, Eq. (15) then gives $\gamma_{s}\left(\vec{r}, \vec{r}^{\prime}\right)$ as a density functional. The equation determining $\theta[n]$ has the form

$$
\nabla^{2} \theta(r)+\frac{\nabla n(r)}{n(r)} \nabla \theta(r)=\lambda \sin [2 \theta(r)],
$$

with $\lambda$ determined by the normalization constraint

$$
\int n(r) \cos ^{2}[\theta(r)] d \vec{r}=1 .
$$

By using Eqs. (15) and (16), it can be shown [10] that, for spherically symmetric, two-level systems (such as the Be atom), Eq. (13) reduces to

$$
z_{s}(r)=4\left[\frac{t_{s}(r)}{r}+\frac{1}{2} \frac{\partial t_{s}(r)}{\partial r}\right] .
$$

As can be seen, a similar formula as for the one-level case has emerged; this is immediately explained by the fact that Eq. (15) gives back the one-level density matrix-density relationship with $\theta(r) \equiv 0$. It must not be assumed, however, that for arbitrary level occupancy (with spherical symmetry), Eq. (18) is general. The Pauli potential will enter explicitly for threeand higher-level occupancy, via Eq. (13). In Eq. (18), $t_{s}(r)$ is determined as a functional of the density through

$$
t_{s}(r)=t_{W}(r)+\frac{1}{2} n(r)[\nabla \theta(r)]^{2},
$$

which is obtained by the insertion of Eq. (15) into Eq. (1) [13].

From Eqs. (6) and (18), we have the result

$-\frac{\partial V(r)}{\partial r}=\frac{4}{n(r)}\left[\frac{t_{s}(r)}{r}+\frac{1}{2} \frac{\partial t_{s}(r)}{\partial r}\right]-\frac{\hbar^{2}}{4 m} \frac{1}{n(r)} \frac{\partial}{\partial r} \nabla^{2} n(r)$,

valid for one- and two-level occupancies, in spherical symmetry. Adding Eqs. (9) and (20), then, yields

$$
\begin{aligned}
& \frac{\hbar^{2}}{2 m} \frac{\vec{r}}{r} \nabla_{\vec{r}}\left[\frac{\nabla_{\vec{r}}^{2} \gamma_{s}\left(\vec{r}, \vec{r}^{\prime}\right)-\nabla_{\vec{r}^{\prime}}^{2} \gamma_{s}\left(\vec{r}, \vec{r}^{\prime}\right)}{\gamma_{s}\left(\vec{r}, \vec{r}^{\prime}\right)}\right]+\frac{4}{n(r)} \\
& \quad \times\left[\frac{t_{s}(r)}{r}+\frac{1}{2} \frac{\partial t_{s}(r)}{\partial r}\right]-\frac{\hbar^{2}}{4 m} \frac{1}{n(r)} \frac{\partial}{\partial r} \nabla^{2} n(r)=0 .
\end{aligned}
$$

Equation (21) formally is an alternative to the March-Suhai equation (4), in the sense that Eq. (21), too, is a differential 
equation for $\gamma_{s}\left(\vec{r}, \vec{r}^{\prime}\right)$ in terms of $n(\vec{r})$ and $t_{s}(\vec{r})$. Through $t_{s}[n]$, it gives a differential equation for the Dirac density matrix in terms of the ground-state density.

Equation (21) can be used to set up a differential equation that contains only an explicit dependence on the density, by eliminating the kinetic energy density from Eq. (4) with the help of Eq. (21). This can be achieved by expressing $t_{s}(r)$ from Eq. (4), and differentiating the resultant equation with respect to $r$. Then $\frac{\partial t_{s}(r)}{\partial r}$ so obtained, and $t_{s}(r)$ expressed from Eq. (4), can be substituted into Eq. (21) to get the desired equation. The result is an important necessary condition for $\gamma_{s}[n]\left(\vec{r}, \vec{r}^{\prime}\right)-$ an explicit differential equation for $\gamma_{s}\left(\vec{r}, \vec{r}^{\prime}\right)$ in terms of $n(\vec{r})$ for one- or two-level occupancy, in the case of spherical symmetry.

\section{GENERALIZATION FOR ARBITRARY LEVEL OCCUPANCY IN ONE DIMENSION}

In one dimension, the appearance of the Pauli potential in the differential virial theorem can be avoided, which leads to a MS-type equation for $\gamma_{s}[n]\left(x, x^{\prime}\right)$ that is valid generally, not just for one- and two-level occupancies. The starting point again is the equation of motion for the Dirac density matrix, in its one-dimensional form,

$$
\frac{\partial^{2} \gamma_{s}\left(x, x^{\prime}\right)}{\partial x^{2}}-\frac{\partial^{2} \gamma_{s}\left(x, x^{\prime}\right)}{\partial x^{\prime 2}}=\frac{2 m}{\hbar^{2}}\left[V(x)-V\left(x^{\prime}\right)\right] \gamma_{s}\left(x, x^{\prime}\right) \text {. }
$$

However, in one dimension, the differential virial theorem takes a much simpler form than Eq. (6) of the threedimensional case [6], the gradient of the single-particle kinetic energy density appearing in the place of the derivatives of the single-particle kinetic energy density tensor with respect to the various position coordinates; that is,

$$
-\frac{\partial V(x)}{\partial x}=\frac{2}{n(x)} \frac{\partial t_{s}(x)}{\partial x}-\frac{\hbar^{2}}{4 m} \frac{n^{\prime \prime \prime}(x)}{n(x)} .
$$

Dividing both sides of Eq. (22) by $\gamma_{s}\left(x, x^{\prime}\right)$ and then differentiating with respect to $x$, we find

$$
\frac{\partial}{\partial x}\left\{\frac{1}{\gamma_{s}\left(x, x^{\prime}\right)}\left[\frac{\partial^{2} \gamma_{s}\left(x, x^{\prime}\right)}{\partial x^{2}}-\frac{\partial^{2} \gamma_{s}\left(x, x^{\prime}\right)}{\partial x^{\prime 2}}\right]\right\}=\frac{2 m}{\hbar^{2}} \frac{\partial V(x)}{\partial x} .
$$

Now we can utilize the differential virial theorem in Eq. (23) to remove $\partial V / \partial x$ from Eq. (24), obtaining

$$
\begin{gathered}
\frac{\partial^{3} \gamma_{s}}{\partial x^{3}}-\frac{\partial^{3} \gamma_{s}}{\partial x \partial x^{\prime 2}}-\gamma_{s}^{-1} \frac{\partial \gamma_{s}}{\partial x}\left(\frac{\partial^{2} \gamma_{s}}{\partial x^{2}}-\frac{\partial^{2} \gamma_{s}}{\partial x^{\prime 2}}\right) \\
+\frac{4 m}{\hbar^{2}} \frac{1}{n(x)} \frac{\partial t_{s}[n](x)}{\partial x} \gamma_{s}-\frac{1}{2} \frac{n^{\prime \prime \prime}(x)}{n(x)} \gamma_{s}=0
\end{gathered}
$$

a nonlinear third-order partial differential equation for the Dirac density matrix as a functional of the ground-state density.

As an elementary check of Eq. (25), the one-level form

$$
\gamma_{s}\left(x, x^{\prime}\right)=\sqrt{n(x)} \sqrt{n\left(x^{\prime}\right)}
$$

for $\gamma_{s}\left(x, x^{\prime}\right)$ can be substituted into it, giving

$$
\begin{aligned}
& \frac{\partial^{3}}{\partial x^{3}} \sqrt{n(x)}-\frac{1}{\sqrt{n(x)}} \frac{\partial}{\partial x} \sqrt{n(x)} \frac{\partial^{2}}{\partial x^{2}} \sqrt{n(x)} \\
& +\frac{4 m}{\hbar^{2}} \frac{1}{n(x)} \frac{\partial t_{s}(x)}{\partial x} \sqrt{n(x)}-\frac{1}{2} \frac{n^{\prime \prime \prime}(x)}{n(x)} \sqrt{n(x)}=0 .
\end{aligned}
$$

In this specific case, $t_{s}(x)$, as a functional of $n(x)$, has the well-known Weizsäcker form [Eq. (10)], and substituting it into Eq. (27), one verifies that it becomes an identity.

We return to general level occupancy, but now with the important example of harmonic confinement $V(x)=x^{2} / 2$; magnetic trapping of ultracold fermion vapors, achieved experimentally by DeMarco and Jin [14], has made low dimensionality experimentally accessible. In the case of harmonic confinement, $t_{s}(x)[n]$ is explicitly known [15] (even in $D$ dimensions [16]) and can be written

$$
t_{s}^{\text {harm }}(x)=n^{3}(x)\left[c_{0}+\frac{1}{12} \int_{0}^{x} \frac{1}{n^{3}\left(x^{\prime}\right)} \frac{d^{3} n\left(x^{\prime}\right)}{d x^{\prime 3}} d x^{\prime}\right],
$$

with $c_{0}=N /\left(3 n^{2}(0)\right)$. With this $t_{s}(x)[n]$, Eq. (25) then achieves the aim of March and Suhai [1] for arbitrary level occupancy for one-dimensional harmonic confinement. Finally, we note that an explicit form for $\gamma_{s}\left(x, x^{\prime}\right)$ has already been written by Husimi [17] for this case. As Lawes and March $[18,19]$ summarized, $\gamma_{s}\left(x, x^{\prime}\right)$ for $N+1$ occupied lowest states is given by

$$
\begin{aligned}
\gamma_{s}\left(x, x^{\prime}\right)= & \frac{1}{2} \phi_{N}(x) \phi_{N}\left(x^{\prime}\right)+\frac{1}{2\left(x-x^{\prime}\right)} \\
& \times\left[\phi_{N}(x) \frac{\partial \phi_{N}\left(x^{\prime}\right)}{\partial x^{\prime}}-\phi_{N}\left(x^{\prime}\right) \frac{\partial \phi_{N}(x)}{\partial x}\right] .
\end{aligned}
$$

When Eq. (29) is substituted into Eq. (25), and the known form for $t_{S}(x)[n]$ is inserted, one obtains an explicit differential equation for the highest occupied orbital $\phi_{N}(x)$ as a functional of the density.

Finally, since in this study, equations stemming from common origins are combined to obtain new ones, the question of independence should be carefully examined. Here, independence of equations should be algebraic independence. An equation determining a functional dependence $f(x)\left[g\left(x^{\prime}\right)\right]$ can be freely combined linearly with its derivative (with respect to $x$ ), for example. To illustrate this, consider the one-particle Schrödinger equation with spherically symmetric Coulombic external potentials, $v(\vec{r})=-Z / r$; this is an equation to determine $\psi(\vec{r})\left[v\left(\vec{r}^{\prime}\right)\right]$ for this special class of potentials. One can differentiate this equation twice, e.g., and can eliminate $Z$ and the energy eigenvalue $E$ by combining the three equations. The result is an equation to be satisfied by $\psi(\vec{r})\left[-Z / r^{\prime}\right]$. (Substituting $\psi(\vec{r})\left[-Z / r^{\prime}\right]$ into that equation will give an identity.) Of course, the new equation does not fully determine $\psi(\vec{r})\left[-Z / r^{\prime}\right]$; it should rather be considered as an important necessary condition for it. As other examples, it can be mentioned that the virial theorem of DFT [20], the differential virial theorem [6,21], and the von Weizsäcker functional [22] can all be obtained (alternatively) via combinations of the Kohn-Sham equations and their derivatives.

In the present study, there are three occasions where the issue of independence may arise: when combining Eq. (9) 
with Eq. (20), Eq. (4) with Eq. (21), and finally, Eq. (23) with Eq. (24). In the first case, independence is ensured by the simple fact that Eq. (9), emerging from the equation of motion for $\gamma_{s}\left(\vec{r}, \vec{r}^{\prime}\right)$, is a generally valid equation, while Eq. (20) is a special form of the differential virial theorem that is valid for one- and two-level spherically symmetric systems only. The fact that Eq. (21) is a third-order differential equation for $\gamma_{s}\left(\vec{r}, \vec{r}^{\prime}\right)$, while Eq. (4) is only a first-order (though highly nonlinear) one, means that the two equations are independent algebraically. The case of Eqs. (23) and (24), however, is different. Not only do they have a common origin [Eq. (22)], but Eq. (23) is a direct consequence of Eq. (24) - the diagonal part of it. Therefore, their combination does not give too much formally. However, $t_{s}(x)$ in Eq. (25) is to be considered as a functional of $n(x), t_{s}[n](x)$, which means that, provided one has an expression for $t_{s}[n](x)$, Eq. (25) gives a necessary condition to be fulfilled by $\gamma_{s}[n]\left(x, x^{\prime}\right)$. That such a situation is real is shown well by the harmonic confinement case mentioned here, where an explicit expression is available for $t_{s}[n](x)$ [Eq. (28)], but $\gamma_{s}[n]\left(x, x^{\prime}\right)$ is unknown.

\section{SUMMARY}

The main achievement of this study is to exhibit in Eq. (21) a differential equation for the Dirac single-particle first-order density matrix in terms of the ground-state electron density and the single-particle kinetic energy density for spherically symmetric electron systems with one- or two-level occupancy, such as the He or the Be atom. With the use of Eq. (21), the kinetic energy density can be eliminated from the March-Suhai equation [1], to which Eq. (21) represents an alternative. The result is a explicit differential equation (a necessary condition) for the Dirac density matrix as a functional of the density. For general level occupancy for three levels and higher, the Pauli potential enters, via Eq. (13). In future work, the Ne atom is well worth exploring, as the next closed-shell, spherically symmetric atom after Be.

In addition to Eq. (21), in Sec. III, the corresponding equation in one dimension, Eq. (25), has been obtained, but this time for arbitrary level occupancy, utilizing the differential virial theorem of one-dimensional systems. As the singleparticle kinetic energy density is already known explicitly as a functional of the density in the case of harmonic confinement, the aim of March and Suhai [1] is achieved for arbitrary level occupancy for this specific one-dimensional case.

The significance of obtaining the Dirac density matrix in terms of the density, on one hand, lies in the fact that from $\gamma_{s}[n]\left(\vec{r}, \vec{r}^{\prime}\right)$, the single-particle kinetic energy and exchange energy density functionals, two essential constituents of density functional theory, can be determined. On the other hand, with the density-matrix functional theory of electron systems gaining stronger and stronger presence in atomic and solid-state physical investigations, it is important to establish connections between the two theories, density-matrix functional theory and traditional DFT.

\section{ACKNOWLEDGMENTS}

NHM wishes to acknowledge that his contribution to this study was brought to fruition during a visit to the University of Antwerp (UA). NHM also wishes to acknowledge his continuing affiliation with UA, made possible by Professors D. Van Dyck and D. Lamoen, and partial financial support by UA through BOF-NOI. IAH would like to acknowledge the hospitality of the ALGC group at VUB.
[1] N. H. March and S. Suhai, Phys. Lett. A 360, 665 (2007).

[2] P. A. M. Dirac, Proc. Cambridge Philos. Soc. 26, 376 (1930); 27, 240 (1931).

[3] R. G. Parr and W. Yang, Density Functional Theory of Atoms and Molecules (Oxford University Press, New York, 1989).

[4] J. C. Slater, Phys. Rev. 81, 385 (1951).

[5] N. H. March, Phys. Rev. A 36, 5077 (1987).

[6] N. H. March and W. H. Young, Nucl. Phys. 12, 237 (1959).

[7] A. Holas and N. H. March, Phys. Rev. A 51, 2040 (1995).

[8] A. Akbari, N. H. March, and A. Rubio, Phys. Rev. A 80, 032509 (2009).

[9] C. F. von Weizsäcker, Z. Phys. 96, 431 (1935).

[10] N. H. March and Á. Nagy, Phys. Rev. A 78, 044501 (2008).

[11] N. H. March, Phys. Lett. A 113, 476 (1986).

[12] M. Levy and A. Görling, Phil. Mag. B 69, 763 (1994).
[13] K. A. Dawson and N. H. March, J. Chem. Phys. 81, 5850 (1984).

[14] B. DeMarco and D. S. Jin, Science 285, 1703 (1999).

[15] N. H. March, P. Senet, and V. E. Van Doren, Phys. Lett. A 270, 88 (2000).

[16] I. A. Howard, N. H. March, and L. M. Nieto, Phys. Rev. A 66, 054501 (2002).

[17] K. Husimi, Proc. Phys. Math. Soc. Jpn. 22, 264 (1940).

[18] G. P. Lawes and N. H. March, J. Chem. Phys. 71, 1007 (1979).

[19] See also I. A. Howard, I. V. Komarov, N. H. March, and L. M. Nieto, J. Phys. A 36, 4757 (2003).

[20] J. C. Slater, J. Chem. Phys. 57, 2389 (1972); J. F. Janak, Phys. Rev. B 9, 3985 (1974).

[21] R. Baltin, J. Chem. Phys. 86, 947 (1987).

[22] T. Gál, J. Phys. B 40, 111 (2007). 